\title{
A wealth of new planetary nebulae from the IPHAS survey
}

\section{Romano L.M. Corradi ${ }^{1,2}$, Laurence Sabin $^{3}$ and the IPHAS collaboration $^{4}$}

${ }^{1}$ Instituto de Astrofísica de Canarias, E-38200 La Laguna, Tenerife, Spain email: rcorradi@iac.es, Isabin@astrosen.unam.mx

${ }^{2}$ Departamento de Astrofísica, Universidad de La Laguna, E-38206 La Laguna, Tenerife, Spain

${ }^{3}$ Instituto de Astronomía, Universidad Nacional Autónoma de México, Apdo. Postal 877, 22800 Ensenada, B.C, Mexico

${ }^{4}$ www. iphas.org

\begin{abstract}
We report on the current status of the search for planetary nebulae (PNe) in the IPHAS survey, the 1800 square degree H-alpha survey of the Northern Galactic Plane carried out at La Palma Observatory. The first major installment of the "IPHAS Catalogue of Extended Nebulae" is presented. This includes a complete census of emission-line nebulae in 320 square degrees toward the inner regions of the Galaxy. In this area, more than 450 emission nebulae have been catalogued including 100 known PNe, and 155 new IPHAS candidate PNe.

A vigorous programme of spectroscopic confirmation of IPHAS candidate PNe over the entire survey area is underway using a number of telescopes worldwide. So far, around one hundred new PNe over the whole IPHAS area have been spectroscopically confirmed. Their main properties, some outstanding examples, as well as the relevance of the IPHAS survey to determine the total PN population in the Galactic disc and its chemical gradient is discussed.
\end{abstract}

Keywords. surveys, Galaxy: disk, planetary nebulae: general

\section{IPHAS overview}

IPHAS is the "INT Photometric H $\alpha$ Survey" of the Northern Galactic Plane. It was was carried out from 2003 to 2009 at the 2.5m Isaac Newton Telescope (INT) of the Observatorio del Roque de Los Muchachos on La Palma, Canary Islands. IPHAS is an international collaboration which involves a number of institutes, mainly from the UK, Spain, the Netherlands, USA and Australia. The aim of the survey is to produce a complete, fully photometric, and spatially detailed $\mathrm{H} \alpha$ map of 1850 square degrees of the northern Galactic Plane between latitudes $-5^{\circ}$ and $+5^{\circ}$

The IPHAS observations are obtained with the Wide Field Camera (WFC) at the INT prime focus. The WFC consists of a mosaic of four $2 \mathrm{k} \times 4 \mathrm{k}$ EEV CCDs, providing a field of view of $34 \times 34 \operatorname{arcmin}^{2}$ with a sampling of $0.33^{\prime \prime}$ per pixel. Images are taken through three filters: a narrow-band $\mathrm{H} \alpha\left(\lambda_{c}=6568 \AA ; \mathrm{FWHM}=95 \AA\right)$ and two broad-band Sloan $r$ and $i$ filters, with matched 120,30 , and 10 s exposures, respectively. In this way, the magnitude range $13 \leqslant r \leqslant 20$ is covered for point sources (the fainter end at $10 \sigma$ ). Pipeline data reduction and data distribution are handled by the Cambridge Astronomical Survey Unit. The main product of the survey is its point sources photometric catalogue, whose Initial Data Release was made available to the community in 2008 (Gonzalez-Solares et al. 2008, http://casu.ast.cam.ac.uk/ag/portal/). The database contains photometry for 80 million sources in the northern Galactic Plane. The basic aspects of IPHAS are 


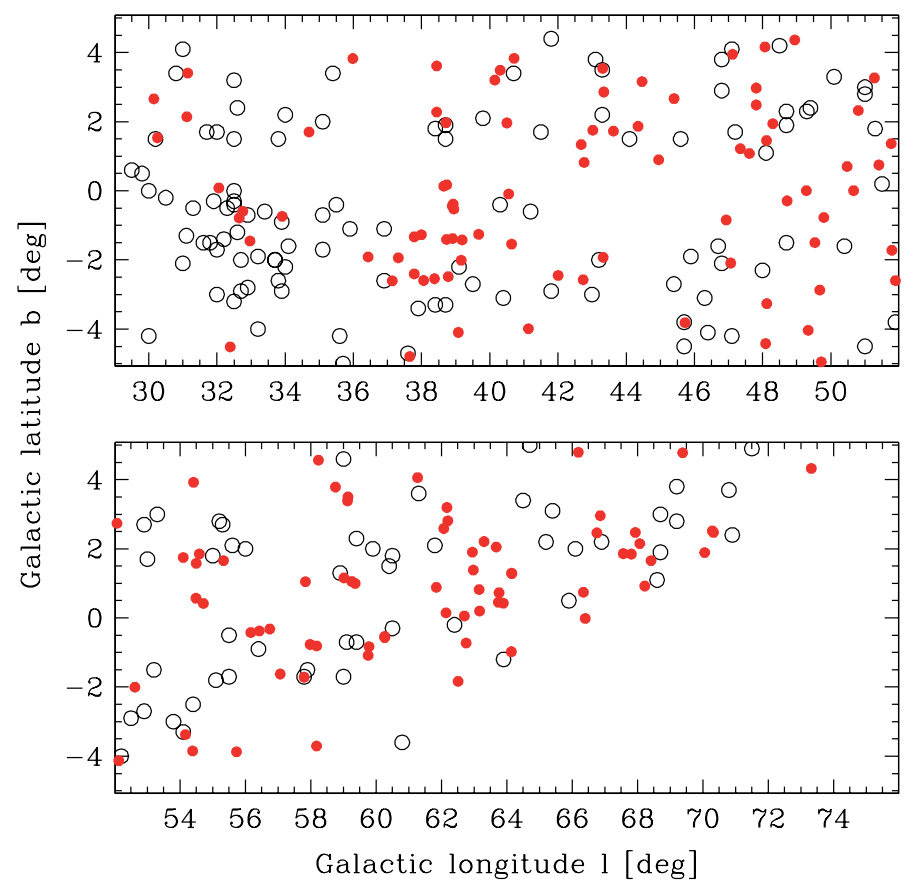

Figure 1. Distribution of the new IPHAS candidate PNe in the region where the search has been completed (full circles) compared to the previously known objects (empty circles).

presented by Drew et al. (2005), and can also be found in the IPHAS home web page at http://www.iphas.org.

\section{IPHAS and the planetary nebulae}

IPHAS presents an opportunity to make a comprehensive search for ionized nebulae, whether compact or extended, in the Northern Galactic Plane. Among them, a complete census of PNe in the Galactic Plane, complementing the results from the MASH survey in the South (Parker et al. 2005), is one of our main goals.

\subsection{Compact nebulae}

Unresolved or small (up to a few arcsec) ionized nebulae are detected by the automatic pipeline which produces the general IPHAS photometric catalogue. The search for such compact PNe is presented by Viironen et al. (2009a,b). Candidates are primarily selected by analysing their colours in the IPHAS colour-colour diagram. Normal PNe appear among the objects with the largest $\mathrm{r}-\mathrm{H} \alpha$ colours, as expected from their almost pure emission-line nature with negligible continuum contribution. Also, they occupy a distinctive position in the new-IR 2MASS colour-colour diagram which was used as a complementary database. However, very interesting PNe can display much smaller $\mathrm{H} \alpha$ line strengths, as well as anomalous 2MASS colours (with remarkable $J-H$ colour excess). This is for instance the case of very young PNe, recently ionized or even in transition from the AGB to the PN phase before they are photoionized, and revealed in IPHAS by the presence of shocked ionized outflows. Or it is the case of very well studied nebulae like M 2-9 or Mz 3, whose real nature as PNe is still the object of debate. Viironen et al. (2009a) discuss four of these complex cases. 


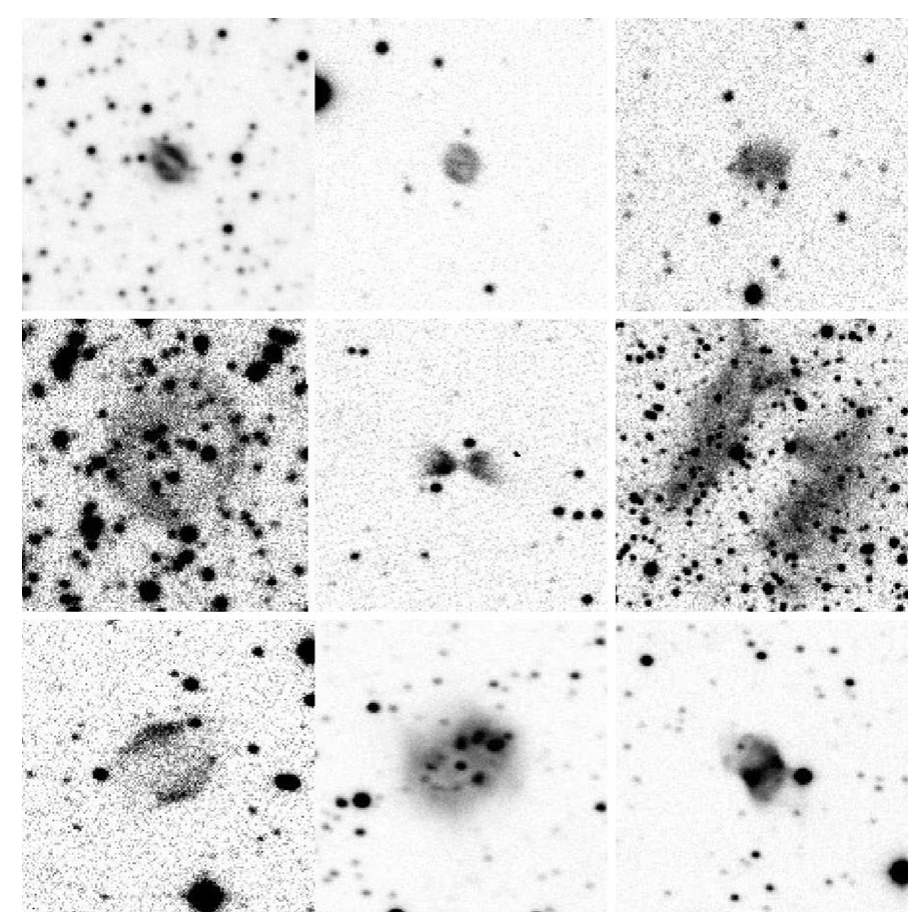

Figure 2. Examples of IPHAS candidates PNe. Each image is $50 \operatorname{arcsec}$ on a side.

\subsection{Extended nebulae: the first release of the IPHAS Catalogue of Extended Nebulae}

In order to find larger nebulae, meticulous and patient visual inspection of the IPHAS images has been undertaken by several people in the IPHAS collaboration, with the help of a number of doctoral and undergraduate students.

The IPHAS area is being scanned at two different binning/resolutions. First, the $\mathrm{H} \alpha$ images are continuum subtracted by properly scaling the $r$ images. Relatively small nebulae are then searched by scanning the IPHAS images after spatial binning of $5 \times 5$ pixels, or $1.6 \times 1.6 \operatorname{arcsec}^{2}$. Large and generally fainter nebulae are searched by producing larger mosaics at $5 \times 5 \operatorname{arcsec}^{2}$ binning. Overlap between these two binning setups, and with the search in the photometric catalogue, assures that we are complete at all sizes and down to a surface brightness level of few $10^{-17} \mathrm{erg} \mathrm{cm}^{-2} \mathrm{~s}^{-1} \operatorname{arcsec}^{-2}$. This is comparable with the sensitivity of the MASH survey.

So far, the search has been completed for the area between $\sim 18 \mathrm{~h} 30 \mathrm{~m}$ and $\sim 20 \mathrm{~h} 00 \mathrm{~m}$ hours in Right Ascension (corresponding to some 350 square degrees of sky), and in progress in the other regions. The area that we have completed contains 450 extended nebulae, of which $51 \mathrm{HII}$ regions and 8 new candidates, 5 SNR and 14 candidates, a few stellar outflows, 80 "diffuse" nebulae (not obviously associated to young stellar environments), and 155 new IPHAS PN candidates. In this same region, $162 \mathrm{PNe}$ were previously known; we have recovered all of them, if the compact PNe retrieved from the photometric catalogue (Viironen et al. 2009b) are added to the 89 extended objects found via visual inspection. Figure 1 shows the distribution of the 155 new candidate PNe and of the previously known objects. This catalogue of IPHAS extended nebulae will be made available to the astronomical community soon (Corradi et al. 2011, in preparation). Figure 2 shows some illustrative example of IPHAS candidate PNe. Most of them are very faint and require $4 \mathrm{~m}$-class or even larger telescopes for deep spectroscopic follow up. 


\subsection{Some highlights}

Wareing et al. (2006) present hydrodynamical modelling of the interaction between the PN Sh 2-188 (one of the largest PNe known, with an extent of 2.8 pc) and the ISM. This is a field to which IPHAS can significantly contribute, owing to its ability to detect faint and large, and thus generally evolved nebulae, where the effects of the interaction between the nebula and the ISM are most developed (see also Sabin et al., these proceedings).

Another main objective of IPHAS is to explore the limits of the Milky Way, as the Galactic anti-centre is best observed from the Northern hemisphere. Several tens of new candidate $\mathrm{PNe}$ have been identified along this direction, and two of them have been studied in detail so far (Mampaso et al. 2006; Viironen et al. 2011).

A remarkable IPHAS discovery is the Necklace PN (Corradi et al. 2011), which has shown to be a key object in the discussion of the formation of asymmetric morphologies, and in particular of equatorial rings and polar jets from post-common envelope binaries.

IPHAS has proven to be very useful also in the study of Galactic novae. Nova Vul 2007 appear particularly interesting, because the IPHAS $\mathrm{H} \alpha$ image revealed the existence of a PN surrounding the nova (Wesson et al. 2008). Its 98-min period makes it the PNe with the binary central star with the shortest orbital period, and a type Ia SN progenitor candidate (Rodríguez-Gil et al. 2010). Among PN related sources, the discovery of a number of S-type stars among the the reddest IPHAS stars (Wright et al. 2008), and the discovery of new symbiotic stars (Corradi et al. 2008, 2010), should also be mentioned.

\section{Perspectives}

The most immediate goal of the study of PNe within IPHAS is to complete the search for extended nebulae over the entire IPHAS area, select candidate PNe according to their morphology and literature data, and obtain follow-up spectroscopy. Spectra will not only allow us to confirm the nature of the nebulae, but also to compute their distance via the IPHAS extinction method (Giammanco et al. 2011) and surface brightness radius (SB-r) technique (Frew \& Parker 2010). Once this is done, we will have a complete sample of PNe, limited in surface brightness, in a specific area of the sky and with known distances. Problems like the 3D distribution in the Galaxy, total PN population size, luminosity function, and their relationships with the properties of individual subclasses of PNe, can be then tackled with the combined IPHAS and MASH samples.

\section{References}

Corradi, R. L. M., Valentini, M., Munari, U., et al. 2010, A\&্A, 509, A41

Corradi, R. L. M., Rodríiguez-Flores, E. R., Mampaso, A., et al. 2008, A\&A, 480, 409

Corradi, R. L. M., Sabin, L., Miszalski, B., et al. 2011, MNRAS, 410, 1349

Drew, J. E., Greimel, R., Irwin, M. J., et al. 2005, MNRAS, 362, 753

Frew, D. J. \& Parker, Q. A. 2010, PASA, 27, 129

Giammanco, C., Sale, S. E., Corradi, R. L. M., et al. 2011, A\&A, 525, A58

Gonzáles-Solares, E. A., Walton, N. A., Greimel, R., et al. 2008, MNRAS, 388, 89

Mampaso, A, Corradi, R. L. M., Viironnen, K., et al. 2006, A\& A, 458, 203

Parker, Q. A., Phillipps, S., \& Pierce, M. J. 2005, MNRAS, 362, 689

Rodríguez-Gi, P. Santander-García, M., Knigge, C., et al. 2008, MNRAS, 407, L21

Viironen, K., Mampaso, A., Corradi, R. L. M., et al. 2009a, A\&A, 502, 113

Viironen, K., Greimel, R., Corradi, R. L. M., et al. 2009b, A\&SA, 504, 291

Viironen, K., Mampaso, A., Corradi, R. L. M., et al. 2011, A\&SA, 530, A107

Wareing, C. E., O'Brien, T. J., Zijlstra, A. A., et al. 2006, MNRAS, 366, 387

Wesson, R., Barlow, M. J., Corradi, R. L. M., et al. 2008, ApJL, 688, L21

Wright, N. J., Greimel, R., Barlow, M. J., et al. 2008, MNRAS, 2390, 929 